\title{
Métabolisme de l'éthanol chez le mouton
}

\author{
A Durix, C Jean-Blain \\ Laboratoire de Nutrition et d'Alimentation, Ecole Nationale Vétérinaire de Lyon, \\ 1, av Bourgelat, BP 83, 69280 Marcy l'Etoile, France
}

Summary - Ethanol metabolism in the sheep was investigated using bolus and constant infusion techniques. Mean half-life of ethanol was $155 \mathrm{~min}$. Ethanol infusion induced a 2-3-fold increase in plasma acetate and propionate levels and a small delayed decrease in that of plasma glucose.

Introduction - Un certain nombre d'aliments (ensilages de mails et d'herbe, pulpes de betteraves, drèches de brasserie) renferment des quantités importantes d'éthanol (de 10 à $40 \mathrm{~g} / \mathrm{kg}$ MS) qui est oxydé en acétate par la microflore du rumen (Pradhan et Hemken, 1970); cependant, des essais en Rusitec nous ont montré que cette voie métabolique n'intéressait que 10 à $25 \%$ de l'éthanol ingéré. Les travaux sur l'utilisation de l'éthanol in vivo chez les ruminants étant très limités, nous avons étudié chez le mouton adulte l'élimination et la transformation de l'éthanol administré par voie veineuse.

Matériel et Méthodes — Un bélier de $55 \mathrm{~kg}$, équipé de deux cathéters chroniques placés dans les veines jugulaires, reçoit du foin à raison de $150 \mathrm{~g} / \mathrm{h}$ pendant les perfusions d'éthanol effectuées selon deux protocoles différents: soit en injection unique : bolus de $20 \mathrm{~g}$, soit en perfusion continue : une dose d'amorçage de $14 \mathrm{~g}$ d'éthanol injectée en $1 \mathrm{~min}$ est suivie d'une perfusion continue $(0,12 \mathrm{~g} / \mathrm{min})$ pendant $5 \mathrm{~h}$. On a effectué 4 répétitions sur le même animal. Des prises de sang sont effectuées régulièrement à l'aide du deuxième cathéter pendant toute la durée de l'expérimentation. Les taux plasmatiques d'éthanol et de glucose sont déterminés par les méthodes enzymatiques classiques, et les acides gras volatils (AGV) par chromatographie en phase gazeuse. Les courbes d'élimina- tion de l'éthanol sont exploitées à l'aide du programme SIPHAR ou FADHA (SIPHAR modifié) (Gomeni, 1984).

Résultats et Discussion - Les figures 1 (a) et (b) indiquent les variations de l'éthanolémie, de la glycémie et des AGV plasmatiques observées respectivement à la suite d'un bolus d'éthanol et d'une perfusion continue. Dans le cas du bolus, la modélisation des résultats par le programme FADHA permet de définir une cinétique à deux compartiments, l'éthanol quittant le compartiment principal par oxydation (cinétique de type michaélien $V_{m}=$ $0,12 \pm 0,04 \mathrm{~g} / \mathrm{h} / \mathrm{l}$ de plasma; $K_{m}=0,48 \pm$ $0,03 \mathrm{~g} / \mathrm{l})$ et par élimination $\left(k_{e}=2,55 \pm\right.$ $0,01)$. Dans ce cas le plasma est presque totalement épuré en $5 \mathrm{~h}$. Une charge de $14 \mathrm{~g}$ suivie d'une perfusion continue de $0,12 \mathrm{~g} / \mathrm{min}$ correspond à la quantité maximale susceptible d'être apportée par l'alimentation. Or on obtient ainsi très rapidement un plateau d'alcoolémie voisin de 1 g/l, correspondant à une clairance de 120 $\mathrm{ml} / \mathrm{min}$, le volume de distribution apparent de l'éthanol étant de 22 I. La demi-vie de l'éthanol mesurée sur la phase décroissante de la perfusion assimilée à une exponentielle est en moyenne de $155 \mathrm{~min}$ (120-187) (programme SIPHAR). 

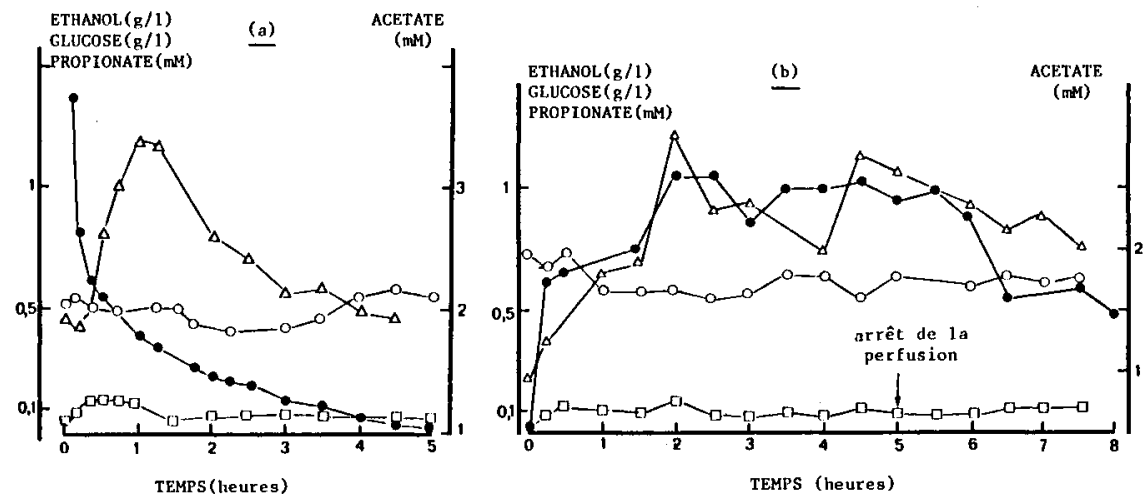

Fig 1. Variations de l'éthanolémie ( - $\rightarrow$ ), de la glycémie $\left(\mathrm{O}_{-} \mathrm{O}\right)$, de l'acétate $(\Delta-\lambda)$ et du propionate $(\square-\square)$ plasmatique : (a) après administration d'un bolus d'éthanol de $20 \mathrm{~g}$ (résultats sur 1 bélier de $55 \mathrm{~kg}$ ); (b) pendant une perfusion continue d'éthanol $(0,12 \mathrm{~g} / \mathrm{min})$ précédée d'un bolus de $14 \mathrm{~g}$ (résultats sur 1 bélier de $55 \mathrm{~kg}$ ).

La concentration du plasma en acétate augmente très rapidement (2 à 3 fois) après la perfusion d'éthanol et atteint une valeur maximale 60 à $120 \mathrm{~min}$ après l'administration du bolus ou le début de la perfusion. Cette élévation traduit la transformation hépatique de l'éthanol en acétate. Le taux plasmatique de propionate habituellement très faible dans le sang périphérique triple précocement et se maintient à une valeur élevée jusqu'à élimination complète de l'éthanol. Par contre une légère baisse de la glycémie apparaît 60 à $90 \mathrm{~min}$ après le début de l'administration d'alcool. Ces deux dernières variations peuvent être interprétées comme une inhibition de la gluconéogenèse hépatique liée à l'augmentation du rapport NADH/NAD+ mitochondrial (Hawkins et Kalant, 1972).

La transformation de l'éthanol en acétate ne semble donc pas être une étape limitante dans l'utilisation métabolique de l'éthanol aux doses où on le rencontre habituellement dans les aliments. L'élévation du taux sanguin d'acétate peut rendre compte de l'élévation du taux butyreux constatée par Pradhan et Hemken (1970). L'incidence pathologique possible liée à une éventuelle dépression de la gluconéogenèse doit être également envisagée.

Pradhan K, Hemken RW (1970) J Dairy Sci 53, 1739-1746

Hawkins RD, Kalant H (1972) Pharmacol Rev 24, 67-157

Gomeni R (1984) Comp Biol Med 14, 25 